\title{
ON VERIFICATION OF FORMAL MODELS OF COMPLEX SYSTEMS
}

\author{
A. A.M. Smyrin ${ }^{1 *}$, E.A. Lukyanova ${ }^{2}$. \\ ${ }^{, 2}$ Lipetsk State Technical University, Russia, V.I. Vernadsky Crimean Federal University, Russia
}

\begin{abstract}
The paper considers the application of the theory of Diophantine equations for the analysis of formal models of complex systems, in particular, Petri component models. For the component-place, the corresponding to this net SLIDE has been examined, its consistency has been established, which means that the network always has the ability to go from one given state to another. After analyzing the work of the $C N$-net, taking into account the work of the component-place, we come to the conclusion that all the listed properties of the constructed model are preserved.
\end{abstract}

Keywords: verification, formal model, complex system

\section{INTRODUCTION}

Investigation of processes and systems of any complexity is effectively carried out by constructing and studying the properties of their models. To implement this approach, it is necessary to build a qualitative model of the system under study. Petri component model ( $\mathrm{CN}$-net) can be used as this model $[1,2]$. Petri component model is an optimal extension of Petri nets (PN) for constructing models of systems with parallelism, characterized by a large number of interacting processes and their considerable dimensionality. The construction of the $\mathrm{CN}$-net allows us to go from the initial detailed model to the simplified description, which guarantees the reliability of the conclusions about the properties of the detailed model of the system under investigation [3].

Subjecting the resulting product to formal analysis using, for example, linear algebra methods [4], we can obtain important information about the structure and behavior of the system being simulated. Many properties of the PN are studied by methods of linear algebra, both static and dynamic ones. In this case, when verifying Petri component models, it is necessary to solve both systems of linear homogeneous Diophantine equations (SLHDE), and systems of linear inhomogeneous Diophantine equations (SLIDE).

The possibilities of verification of $\mathrm{CN}$-models of complex systems are considered in the work on the model of the railway station with the use of SLHDE for finding of $S$ - and $T$ - invariants of a given model as well as SLIDE - for establishing the possibility of functioning of the subnet (componentplace) of the $C N$-model.

\section{PETRI MODEL ANALYSIS TOOLS}

For PN, $N=\left(S, W, M_{0}\right)$ (where $S=(P, T, F)$ is net, $W: F \rightarrow N$ - function of the multiplicity of the arcs, $F \subseteq P \times T \cup T \times P$ - the incidence relation, $M_{0}$ - initial markup of the net) with $m$ places and $n$ transitions, a matrix of incidence $A$ is constructed, sized $m \times n$ with integer coefficients $a_{i j}=I\left(t_{j}, p_{i}\right)-I\left(p_{i}, t_{j}\right)$, where $I$ is the incidence function of this $\mathrm{PN}$, and the coefficients $a_{i j}$ represent the number of tokens that move, change and are added to the location $p_{i}$ when the transition $t_{j}$ occurs in the net $N$. According to the incidence matrix $A$, a system of equations of states of a given $\mathrm{PN}$ is constructed 
$A x=d$,

where $d=M_{k}-M_{0}\left(M_{0}, M_{k}-\right.$ respectively, the initial and final grid layouts of net $\left.N\right)$, $x=\sum_{j=1}^{k} u_{j}$, where $u_{k}$ - control vector consisting of $n-1$ zero components and one $j$ component equal to 1 , signaling the operation at the $k$ step of the transition $t_{j}$. If the markup $M_{k}$ is reachable from the markup $M_{0}$, the system (1) always has a solution [5].

The solution $x$ of SLHDE $A x=0$, when in (1) $M_{k}=M_{0}$, is called $T$-invariant of the net $N$. The solution $y$ SLHDE $A^{T} y=0$ is called $S$-invariant of the net $N$.

The calculation of $S$ - и $T$-invariants for a given PN makes it possible to verify such properties as reachability, boundedness, repeatability, conservatism, consistency. A special role in solving the problems of boundedness is played by minimal generating sets of $S$ - and $T$-invariants [6]. The generating set of $S$-invariants ( $T$-invariants) is called the minimal generating set of $S$-invariants ( $T$ invariants), if there is no non-empty subset of it, which is also a generating set.

It follows from the theory of Diophantine equations $[5,6,7]$, that the truncated set of solutions of SLHDE, corresponding to a given PN, is the minimal generating set of $S(T)$-invariants, to construct this set one can use the TSS -algorithm [8].

To establish the compatibility of the SLIDE, the compatibility criterion of the SLIDE is used [7].

Theorem 1. A system $S$ of linear inhomogeneous Diophantine equations is compatible if and only if the auxiliary system $S^{*}$ is compatible.

For SLIDE

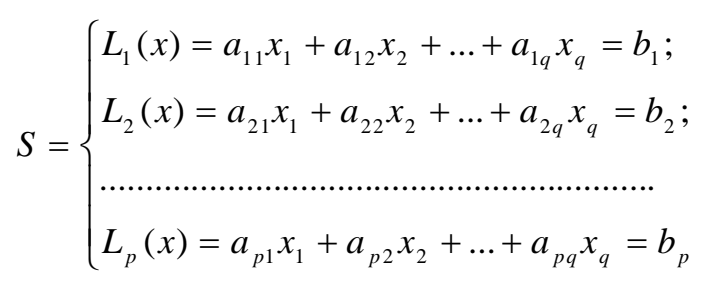

auxiliary system $S^{*}$ has the form

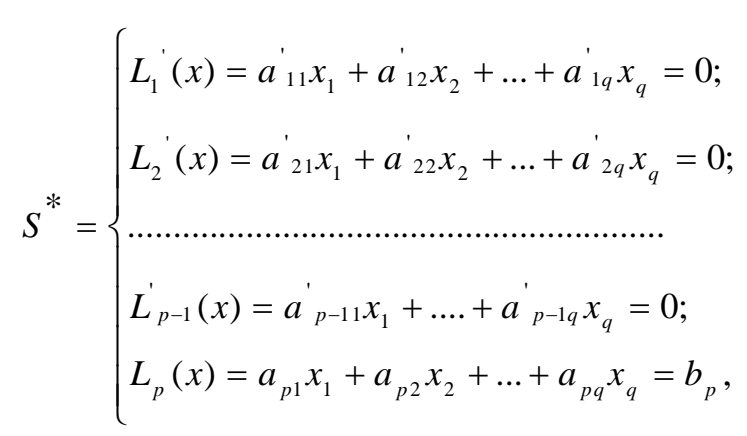


where $b_{p}>0$, and the first $p-1$ equations are obtained as linear combinations:

for all $i=1 \partial o p-1$, if $b_{i}>0$, then $L_{i}^{\prime}=b_{i} L_{p}(x)-b_{p} L_{i}(x), \quad$ if $\quad b_{i}<0$, then $L_{i}^{\prime}=-b_{i} L_{p}(x)+b_{p} L_{i}(x)$.

Theorem 2. A system $S^{*}$ is compatible if and only if equation

$d_{1} u_{1}+d_{2} u_{2}+\ldots+d_{k} u_{k}-t b_{p}=0$

has at least one solution $\left(\alpha_{1}, \alpha_{2}, \ldots, \alpha_{k}, \gamma\right)$ in a set $N$ such that the coordinates of the vector $v=\alpha_{1} e_{1}^{\prime}+\alpha_{2} e_{2}^{\prime}+\ldots+\alpha_{k} e_{k}^{\prime}$ are a multiple of the number $\gamma$. Here $d_{1}, d_{2}, \ldots, d_{k}$ are the values $L_{p}(x)$ on the vectors $e_{1}^{\prime}, e_{2}^{\prime}, \ldots, e_{k}^{\prime}$, that are vectors of the subsystem TSS of the first $p-1$ equations of the system $S^{*}, t \neq 0, \gamma \neq 0, \alpha_{i}, t, \gamma \in N, i=1$ до $k$.

Let's consider a system $S$, in which the equation with a free term $b_{p}=1$ can be chosen as the equation $L_{p}(x)$. If for the first $p-1$ equations of the system $S^{*}$ it is possible to construct TSS, then by Theorem 2 it is necessary to find at least one solution of equation

$d_{1} u_{1}+d_{2} u_{2}+\ldots+d_{k} u_{k}-t=0$

to establish the compatibility of the system $S^{*}$, satisfying the hypothesis of the theorem.

Let any two coefficients $d_{i}$ and $d_{j}$ of the last equation be mutually prime numbers of opposite signs, then assigning to all $u_{r}(r=1,2, \ldots k, r \neq i, j)$ and a $t$ value 1 , and using the Bezout relation for $d_{i}$ and $d_{j}$ and the extended Euclidean algorithm, we find $u_{i}$ and $u_{j}$. Thus, the desired solution of the last equation will be constructed.

The following condition is obtained for establishing the compatibility of the SLIDE.

Theorem 3. If there is an equation $L_{p}(x)$ with a free term equal to 1 in the SLIDE, and if at least two values $L_{p}(x)$ on the vectors $T S S$ of the first $p-1$ equations of the system $S^{*}$ are mutually prime numbers with opposite signs, then such a system is compatible.

\section{ANALYSIS OF THE PETRY MODEL}

Let's consider a Petri net from [9], shown in Fig. 1, a). This net simulates the movement of trains through some dead-end railway station, with one input track and three internal tracks. In Fig. 1, b) the model is presented as a $C N$-net. In this $C N$-net, the place $P_{2}{ }^{*}$ is a component-place, which is separately shown in Fig. 1, c).

Having written the incidence matrix for the $C N$-net and established, that for this net $M_{k}=M_{0}=(1,0,1)$, we build the SLHDE $A x=0$. Its positive integer solutions are $T$-invariants of $C N$-net. The problem of the compatibility of the resultant SLHDE is solved by constructing a truncated set of solutions by an TSS -algorithm. The result is a vector $e=(1,1,1)-T$-invariant. To 
search for the $S$ - invariants we construct a SLHDE $A^{T} y=0$, the compatibility of which is also established by the TSS -algorithm. The result are vectors $e_{1}=(0,0,1),, e_{2}=(1,1,0)$.

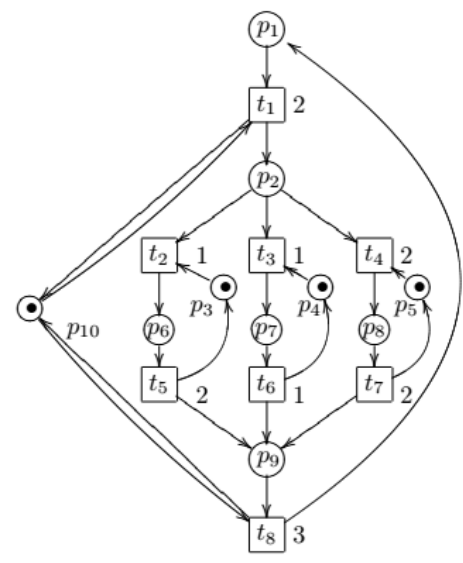

a

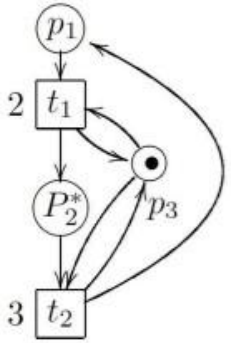

b

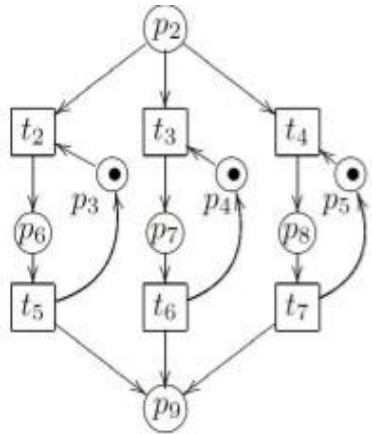

$\mathrm{c}$

Fig. 1. Models: a) a dead-end railway station in the form of PN; b) a dead-end railway station in the form of a $\mathrm{CN}$-net; c) component-place $P_{2}{ }^{*}$

For the net that displays the component-place $P_{2}{ }^{*}$, the initial markup $M_{0}=(1,1,1,1,0,0,0,0)$ and the markup at the $k$ - step $M_{k}=(0,1,1,1,0,0,0,1)$ do not coincide, so we build the SLIDE $S$ и and its corresponding auxiliary system $S^{*}$.

$$
S=\left\{\begin{array}{l}
-x_{1}-x_{2}-x_{3}=-1 ; \\
-x_{1}+x_{4}=0 ; \\
-x_{2}-x_{5}=0 ; \\
-x_{3}+x_{6}=0 ; \\
x_{1}-x_{4}=0 ; \\
x_{2}-x_{5}=0 \\
x_{3}-x_{6}=0 ; \\
x_{4}+x_{5}+x_{6}=1
\end{array} \quad S^{*}=\left\{\begin{array}{l}
-x_{1}-x_{2}-x_{3}+x_{4}+x_{5}+x_{6}=0 \\
-x_{1}+x_{4}=0 \\
-x_{2}-x_{5}=0 \\
-x_{3}+x_{6}=0 \\
x_{1}-x_{4}=0 ; \\
x_{2}-x_{5}=0 \\
x_{3}-x_{6}=0 ; \\
x_{4}+x_{5}+x_{6}=1
\end{array}\right.\right.
$$

Vectors $\grave{e_{1}}=(1,0,0,1,0,0), \grave{e_{2}}=(0,0,1,0,0,1)$ are TSS subsystems, comprised of the first 7 equations. Values $L_{8}(x)$ on vectors TSS: $L_{8}\left(\grave{e}_{1}\right)=1, L_{8}\left(\grave{e}_{2}\right)=1$. Theorem 3 is not applicable. According to Theorem 2, we obtain the equation $1 u_{1}+1 u_{2}-1 t=0$. Let's take one of its roots, for example, $(1,0,1)$. Then, the vector-solution of the system $S^{*}$ has the form $1 \grave{e}_{1}+0 \grave{e}_{2}=1(1,0,0,1,0,0), S^{*}$ is consistent. By Theorem 1, the system $S$ is compatible to the corresponding system $S^{*}$.

\section{CONCLUSION}


The analysis of the constructed Petri nets allows us to establish the properties of the formal model. Thus, from the set of $S$-invariants $e_{1}=(0,0,1),, e_{2}=(1,1,0), C N$-network of the dead-end railway station it is clear that all its places are covered by non-zero coordinates, this means that the network is limited.

You can get an upper score for tokens that fit anywhere, for example, for a place $P_{2}{ }^{*}$ : $M\left(P_{2}{ }^{*}\right) \leq \frac{M_{0} e_{1}^{T}}{e_{1}\left(P_{2}^{*}\right)}=1$ The same score for the number of tokens can be obtained for the rest of the $C N$ net in question. This means that the considered net is safe.

The presence of $T$-invariants indicates the possession of the net by the property of repeatability, which is interpreted as the constant movement of trains through the station. The net has the consistency property, because there is a sequence of transitions triggered on the net, which starts at least once, which can be interpreted for the station as the departure of trains.

For the component-place, the corresponding to this net SLIDE has been examined, its consistency has been established, which means that the network always has the ability to go from one given state to another.

After analyzing the work of the $\mathrm{CN}$-net, taking into account the work of the component-place, we come to the conclusion that all the listed properties of the constructed model are preserved.

\section{ACKNOWLEDGEMENTS}

The reported study was funded by RFBR according to the research project № 16-07-00854 a.

\section{REFERENCES}

E. Lukyanova Component modeling: on connections of detailed Petri model and component model of parallel distributed system //ITHEA, 2013. P. 15-22.

E.A. Lukyanova The structural elements of a Petri net component //Problemy programuvannya. 2012. No. 2-3. P. 25-32.

E.A. Lukyanova, A.V. Dereza The study. Of the structural elements of the single-type CN-network during the component modeling and analysis of complex systems with concurrency //Cybernetics and systems analysis. 2012. No. 6. P.20-29.

T. Murata Petri Nets: Properties, Analysis and Applications //Proceedings of the IEEE. 1989. Vol. 77. No. 4. P. 541-580.

S.L. Kryviy Algorithms for computation of systems of linear Diophantine equations in integer domains //Cybernetics and systems analysis. 2006. No. 2. P.3-17.

S.L. Kryviy. On some methods of computation and compatibility criteria for systems of linear Diophantine equations in the region of natural numbers. //Cybernetics and systems analysis.

1999. No. 4. P.12-36.

S.L. Kryviy. A compatibility criterion for systems of linear Diophantine equations over the set of natural numbers. // Cybernetics and systems analysis. 1999. No. 5. P.107-112.

S.L. Kryviy On the calculation of the minimal set of invariants of Petri nets. //Artificial Intelligence. 2001. No. 3. P.199-206.

A.M. Shmyrin., I.A. Sedykh, E.A. Lukyanova On the representation of the Petri component net by a neighborhood system. I/Vestnik of LSTU. 2016. No. 4 (30). P.35-40. 